\title{
OPEN Enzyme systems involved in glucosinolate metabolism in Companilactobacillus farciminis KB1089
}

Hiroko Watanabe ${ }^{1,2,5}$, Riku Usami $^{1,5}$, Shigenobu Kishino ${ }^{1,5}, K^{1}$ Kengo Osada ${ }^{1}$, Yudai Aoki ${ }^{3}$ Hironobu Morisaka ${ }^{1}$, Masatomo Takahashi ${ }^{4}$, Yoshihiro Izumi ${ }^{4}$, Takeshi Bamba ${ }^{4}$, Wataru Aoki ${ }^{1}$, Hiroyuki Suganuma ${ }^{3}$ \& Jun Ogawa ${ }^{1 凶}$

Cruciferous vegetables are rich sources of glucosinolates (GSLs). GSLs are degraded into isothiocyanates, which are potent anticarcinogens, by human gut bacteria. However, the mechanisms and enzymes involved in gut bacteria-mediated GSL metabolism are currently unclear. This study aimed to elucidate the enzymes involved in GSL metabolism in lactic acid bacteria, a type of gut bacteria. Companilactobacillus farciminis KB1089 was selected as a lactic acid bacteria strain model that metabolizes sinigrin, which is a GSL, into allylisothiocyanate. The sinigrin-metabolizing activity of this strain is induced under glucose-absent and sinigrin-present conditions. A quantitative comparative proteomic analysis was conducted and a total of 20 proteins that were specifically expressed in the induced cells were identified. Three candidate proteins, $\beta$-glucoside-specific IIB, IIC, IIA phosphotransferase system (PTS) components (CfPttS), 6-phospho- $\beta$-glucosidase (CfPbgS) and a hypothetical protein ( $C f$ NukS), were suspected to be involved in sinigrin-metabolism and were thus investigated further. We hypothesize a pathway for sinigrin degradation, wherein sinigrin is taken up and phosphorylated by CfPttS, and subsequently, the phosphorylated entity is degraded by $C f \mathrm{PbgS}$. As expression of both $p t t S$ and $p b g S$ genes clearly gave Escherichia coli host strain sinigrin converting activity, these genes were suggested to be responsible for sinigrin degradation. Furthermore, heterologous expression analysis using Lactococcus lactis suggested that CfPttS was important for sinigrin degradation and $C f \mathrm{PbgS}$ degraded phosphorylated sinigrin.

Isothiocyanates (ITCs) are bioactive functional food factors that exert anti-oxidative, anti-inflammatory and anticarcinogenic effects and induce a host-detoxifying defense system ${ }^{1-5}$. ITCs are derived from glucosinolates (GSLs), which have a non-bioactive thioglucoside bond and are present in these cruciferous vegetables such as broccoli ${ }^{6}$. Their production involves hydrolysis of the $S$-glycosidic linkage in GSLs by human gut bacteria ${ }^{7}$ as common in the metabolism of other glycosides ${ }^{8}$. Thus, with gut bacteria mainly responsible for the beneficial physiological effects of ITCs upon GSL intake, there is a great interest in the determination of how human gut bacteria can degrade GSLs. Some lactic acid bacteria such as Ligilactobacillus agilis $\mathrm{R}_{1} 6^{9}$ and Lactococcus lactis subsp. lactis $\mathrm{KF} 147^{10}$ have exhibited sinigrin (allyl- $\beta$-glucosinolate) degradation. Since lactic acid bacteria exist in intestinal tract and in fermented vegetables, they probably play important roles in GSLs-degradation and ITCs-generation in the process of digestion and fermentation, respectively. However, the corresponding enzymes remained to be elucidated.

We isolated Companilactobacillus farciminis KB1089 with notable sinigrin-degrading and allylisothiocyanate (AITC)-generating activities (Fig. 1A) from Japanese traditional-vegetable pickles. In this paper, we describe identification of the genes responsible for sinigrin degradation to AITC in Cb. farciminis KB1089 by quantitative

${ }^{1}$ Division of Applied Life Sciences, Graduate School of Agriculture, Kyoto University, Kitashirakawaoiwake-cho, Sakyo-ku, Kyoto 606-8502, Japan. 'Laboratory for Circular Bioeconomy Development, Office of Society Academia Collaboration for Innovation, Kyoto University, Kitashirakawaoiwake-cho, Sakyo-ku, Kyoto 606-8502, Japan. ${ }^{3}$ Nature and Wellness Research Department, Innovation Division, KAGOME CO. LTD., 17 Nishitomiyama, Nasushiobara-shi, Tochigi 329-2762, Japan. ${ }^{4}$ Division of Metabolomics, Medical Institute of Bioregulation, Kyushu University, 3-1-1 Maidashi, Higashi-ku, Fukuoka 812-8582, Japan. ${ }^{5}$ These authors contributed equally: Hiroko Watanabe, Riku Usami and Shigenobu Kishino. ${ }^{\circledR}$ email: ogawa@kais.kyoto-u.ac.jp 
A<smiles>C=CCN=C=S</smiles>

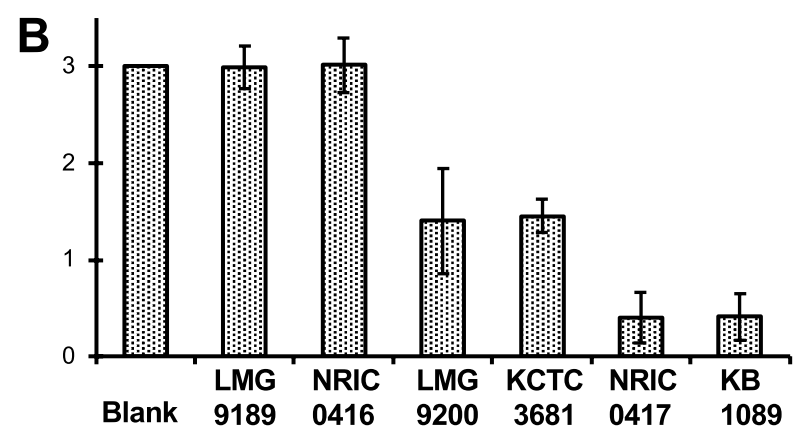

Figure 1. Screening of glucosinolate (GSL)-degrading lactic acid bacteria. (A) Structures of the substrate (sinigrin) and product (allyl isothiocyanate, AITC). (B) Amounts of residual sinigrin in the culture medium (initial sinigrin; $3.0 \mathrm{mM}$ ) after $24 \mathrm{~h}$ of cultivation of each strain of $\mathrm{Cb}$. farciminis. Blank indicates the uninoculated sample. Data represent mean values $(n=3)$. Error bars indicate $95 \%$ confidence interval $(C I)$.

comparative proteomic analysis. Heterologous expression and functional analysis of these genes first confirmed the involvement of a coupled action by $\beta$-glucoside-specific IIB, IIC, IIA phosphotransferase system (PTS) components $(C f \mathrm{PttS})$ and 6-phospho- $\beta$-glucosidase $(C f \mathrm{PbgS})$ in sinigrin degradation by lactic acid bacteria.

\begin{abstract}
Results
Screening of sinigrin-degrading lactic acid bacteria. We performed screening of sinigrin-degrading bacteria among 279 strains, including plant associated lactic acid bacteria and animal gut bacteria. Only four strains exhibited sinigrin-degrading activity during cultivation in the sinigrin-containing culture medium (S3-mMRS). The lactic acid bacteria strain, KB1089, isolated from pickled Japanese turnips, exhibited the highest sinigrin-degrading activity. This strain was identified as Companilactobacillus farciminis via 16S rRNA and pheS gene sequencing. Then, sinigrin-degrading activities of another five strains of $\mathrm{Cb}$. farciminis available in public culture collections were evaluated. Cb. farciminis LMG9200, KCIC3681 and NRIC0417 exhibited sinigrin-degrading activity, whereas LMG9189 and NRIC0416 exhibited no activity (Fig. 1B). Thus, the sinigrindegrading phenotype appeared to be strain-specific. Strain KB1089 exhibited the highest activity and was used for further investigation.
\end{abstract}

Sinigrin-degrading activity of Cb. farciminis KB1089. The strain KB1089 degraded sinigrin only after complete glucose consumption coupled with OD increase and $\mathrm{pH}$ decline during the cultivation in G10S6mMRS medium containing sinigrin and glucose (Fig. 2A). The cultivated cells in the sinigrin-containing medium (G3S3-mMRS) showed sinigrin-degrading and AITC-producing activities (Fig. 2B, Supplementary Fig. S1). On the other hand, the cultivated cells in the sinigrin-free medium (G3-mMRS medium) showed no sinigrin-degrading activity (Fig. 2B). This indicated that the sinigrin-degrading activity of Cb. farciminis KB1089 was induced by sinigrin.

Comparative proteomics in sinigrin-induced and non-induced cells of $\mathrm{Cb}$. farciminis KB1089. The proteins expressed in the sinigrin induced cells and in the non-induced cells were digested and applied to nano-liquid chromatography-tandem mass spectrometry (LC-MS/MS). The bottom-up proteomics analysis of the results revealed the amino acid sequences of the sinigrin-induced proteins. A total of 20 proteins were specifically expressed in the induced cells (Table 1). Among the 20 identified proteins, we selected 3 proteins: $\beta$-glucoside-specific IIB, IIC, IIA PTS component (CfPttS), 6-phospho- $\beta$-glucosidase $(C f \mathrm{PbgS})$ and a hypothetical protein $(C f N u k S)$ for further analysis based on the following observations: The three genes fig|1612.50.peg.2357, fig|1612.50.peg.2356 and fig|1612.50.peg.2355 encoding CfPttS, CfPbgS and CfNukS, respectively, existed adjacent to each other in the Cb. farciminis KB1089 genome (Table 1, Fig. 3A); The homologous genes were present in the genomes of the other sinigrin-degrading strains of $\mathrm{Cb}$. farciminis (strains LMG9200, KCTC3681 and NRIC0417); No homologous genes were found in the genomes of the strains of $\mathrm{Cb}$. farciminis without the sinigrin-degrading activity (the strains LMG9189 and NRIC0416).

Protein analysis using InterPro (https://www.ebi.ac.uk/interpro/) revealed the following results: CfPttS contains three structurally distinct domains, IIA, IIB and $\mathrm{IIC}^{11}$, which form a membrane-bound complex; CfPbgS 
A

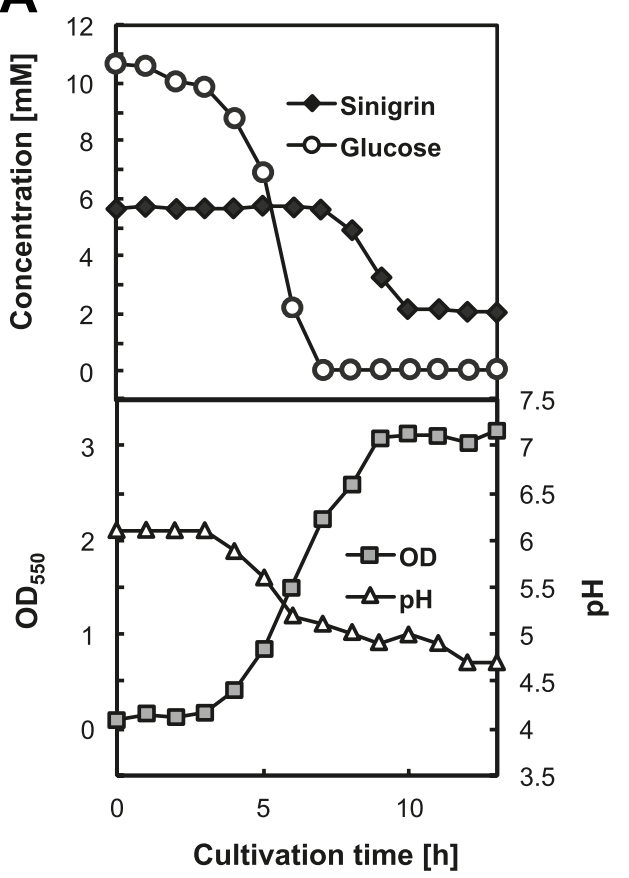

B

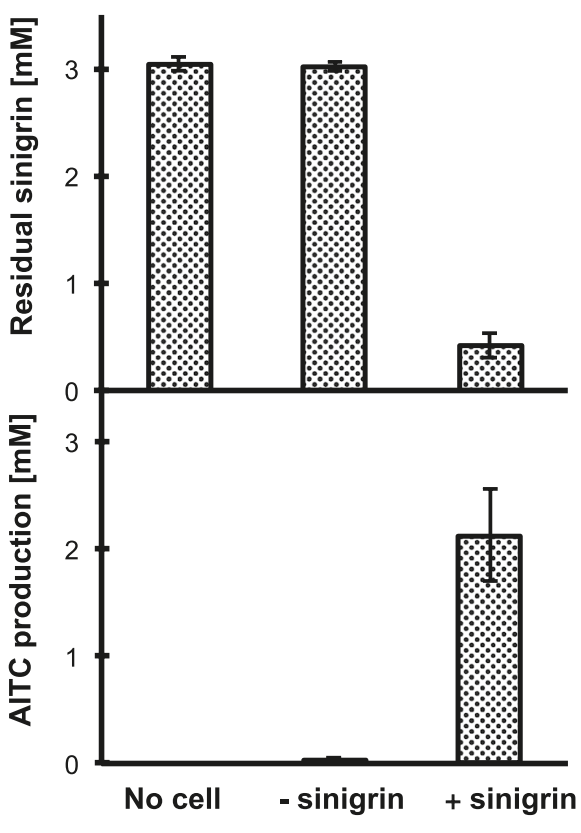

Figure 2. Sinigrin-degrading activity of $C b$. farciminis KB1089. (A) Profiles of glucose and sinigrin concentrations, optical density $\left(\mathrm{OD}_{550}\right)$ and $\mathrm{pH}$ during $\mathrm{Cb}$. farciminis $\mathrm{KB} 1089$ cultivation in the G10S6-mMRS medium. (B) Amounts of residual sinigrin (upper graph) and produced AITC (lower graph) in the resting cell reaction mixtures using washed cells obtained after glucose consumption cultivated in G3-mMRS medium (-sinigrin) or G3S3-mMRS medium (+sinigrin). 'No cell' indicates a reaction solution without cells. Data represent mean value $(n=3)$. Error bars indicate $95 \%$ CI.

belongs to the GH1 family ${ }^{12}$; and CfNukS contains a phosphate-binding loop (P-loop), which is a common motif in ATP-binding proteins ${ }^{13}$ (Table S4). The PTS is a major machinery for importing sugars into the cell with simultaneous phosphorylation of the sugars, which initiate sugar metabolisms in lactic acid bacteria ${ }^{11}$. CfPttS and $C f \mathrm{PbgS}$ appeared to be involved in sugar metabolism initiated by the PTS. We hypothesized the existence of a sinigrin-degrading pathway in Cb. farciminis KB1089 based on the PTS-mediated pathway (Fig. 3B). In the hypothetical pathway, sinigrin is imported to the cytosol, and its glucose moiety is phosphorylated by CfPttS. The phosphate group may be transferred from phosphoenolpyruvate (PEP) via the universal PTS components, Enzyme I (EI) and histidine-containing phosphocarrier protein $(\mathrm{HPr})^{11}$, and $\mathrm{CfPttS}$ to sinigrin. Subsequently, the $S$-glycosidic linkage of phosphorylated sinigrin is hydrolyzed by $C f \mathrm{PbgS}$, followed by a nonenzymatic rearrangement and sulfate group elimination to yield $\mathrm{AITC}^{14}$.

Heterologous expression and functional analysis of candidate proteins. The functions of the genes ( $p t t S, p b g S$ and $n u k S$ ) were evaluated through the heterologous expression in E. coli Rosetta 2 (DE3) and $L c$. lactis NZ9000. None of the E. coli strains harboring empty vectors showed target activity. The strains harboring one candidate gene (pET28-pttS, pET-28-pbgS, and pET28-nukS), or a combination of two genes, namely, $p t t S$ and $n u k S$ (pRSF-pttS-nukS), or pbgS and nukS (pET21-pbgS/pRSF-nukS), showed no target activity. The two E. coli transformants that harbored both $p t t S$ and $p b g S$ genes clearly demonstrated sinigrin-degrading activity (i.e. E. coli pET21-pbgS/pRSFDuet-pttS-nukS and E. coli pET21-pbgS/pRSFDuet-pttS) (Fig. 4A). These strains exhibited a similar extent of target activity (Fig. 4A). Therefore, we concluded that CfPttS and CfPbgS are probably the enzymes responsible for sinigrin degradation. However, the function of the hypothetical protein (CfNukS) remains to be elucidated.

The Lc. lactis NZ9000 transformants harboring only the nukS gene (Lc. lactis pNZ7021-nukS) or the pbgS gene (Lc. lactis pNZ7021-pbgS) degraded sinigrin into AITC the same extent as Lc. lactis pNZ7021. However, the strains harboring only the $p t t S$ gene (Lc. lactis pNZ7021-pttS) and all three candidate genes (Lc. lactis pNZ7021$p t t S$-pbgS-nukS) exhibited higher sinigrin-degrading activity (Fig. 4B). These strains exhibited the same extent activities (Fig. 4B).

Two genes encoding CfPbgS homologues were found in Lc. lactis NZ9000 through a Basic Local Alignment Search Tool (BLAST) search. One of these genes exists adjacent to a gene encoding a CfPttS homologue (Fig. 5). It is assumed that the sinigrin-degrading activity of the vector control strain is caused by these homologues. Since the exogenous gene expression of $C f \mathrm{PttS}$ led to an increase in activity, transportation and phosphorylation have been considered as significant processes for sinigrin degradation in Lc. lactis NZ9000. 


\begin{tabular}{|c|c|c|c|c|c|}
\hline Protein accession in the proteomic data set & Coverage & Score Mascot & \#PSMs & Gene accession (PATRIC ID) & Gene description \\
\hline fig|6666666.361936.peg.193 & 30.0 & 50.6 & 3 & fig|1612.50.peg.18 & Copper-transporting ATPase \\
\hline fig|6666666.361936.peg.624 & 9.62 & 36.6 & 1 & fig|1612.50.peg.198 & hypothetical protein \\
\hline fig|6666666.361936.peg.592 & 8.51 & 37.7 & 1 & fig|1612.50.peg.230 & Putative regulator of the mannose operon, $\mathrm{ManO}$ \\
\hline fig|6666666.361936.peg.125 & 24.1 & 28.5 & 1 & fig|1612.50.peg.450 & hypothetical protein \\
\hline fig|6666666.361936.peg.2171 & 17.3 & 42.3 & 1 & fig|1612.50.peg.1020 & hypothetical protein \\
\hline fig|6666666.361936.peg.2179 & 6.78 & 32.7 & 2 & fig|1612.50.peg.1028 & Hydroxymethylglutaryl-CoA reductase \\
\hline fig|6666666.361936.peg.1153 & 4.03 & 25.0 & 1 & fig|1612.50.peg.1081 & Autolysin, amidase \\
\hline fig|6666666.361936.peg.2477 & 6.47 & 33.5 & 2 & fig|1612.50.peg.1254 & hypothetical protein \\
\hline fig|6666666.361936.peg.373 & 40.7 & 429.2 & 24 & fig|1612.50.peg.1329 & NAD-dependent protein deacetylase of SIR2 family \\
\hline fig|6666666.361936.peg.372 & 28.2 & 216.2 & 12 & fig|1612.50.peg.1330 & NAD-dependent protein deacetylase of SIR2 family \\
\hline fig|6666666.361936.peg.1002 & 10.7 & 96.0 & 3 & fig|1612.50.peg.1594 & 4-hydroxy-tetrahydrodipicolinate synthase \\
\hline fig|6666666.361936.peg.2091 & 27.3 & 88.7 & 6 & fig|1612.50.peg.1699 & Regulatory protein Spx \\
\hline fig|6666666.361936.peg.2480 & 30.4 & 28.7 & 3 & fig|1612.50.peg.1810 & Thioredoxin \\
\hline fig|6666666.361936.peg.1539 & 4.67 & 26.6 & 1 & fig|1612.50.peg.1854 & Ribonuclease HII \\
\hline fig|6666666.361936.peg.1547 & 7.53 & 118.7 & 2 & fig|1612.50.peg.1862 & Thymidylate synthase \\
\hline fig|6666666.361936.peg.1436 & 16.7 & 51.7 & 1 & fig|1612.50.peg.2065 & SSU ribosomal protein S21p \\
\hline fig|6666666.361936.peg.1787 & 2.62 & 29.8 & 2 & fig|1612.50.peg.2257 & ATP synthase F0 sector subunit c \\
\hline fig|6666666.361936.peg.1336 & 25.1 & 405.8 & 21 & fig|1612.50.peg.2355 & hypothetical protein \\
\hline fig|6666666.361936.peg.1335 & 6.69 & 104.0 & 5 & fig|1612.50.peg.2356 & 6-phospho- $\beta$-glucosidase \\
\hline fig|6666666.361936.peg.1333 & 6.69 & 38.9 & 1 & fig|1612.50.peg.2357 & $\begin{array}{l}\text { PTS system, } \beta \text {-glucoside-specific IIB component/PTS } \\
\text { system, } \beta \text {-glucoside-specific IIC component/PTS system, } \\
\beta \text {-glucoside-specific IIA component ( } \beta \text {-glucoside-specific } \\
\text { IIB, IIA, IIC PTS components) }\end{array}$ \\
\hline
\end{tabular}

Table 1. Proteins detected only in induced cells of Companilactobacillus farciminis KB1089. The data were filtered with cut-offs Score Mascot $>0$ and q-value $\leq 0.01$, corresponding to a $1 \%$ false discovery rate on a spectral level. Protein accession numbers are available in the data set deposited in jPOST with accession number PXD011820. The numbers of peptides spectrum matches (\#PSMs) reflect approximate amounts of detected proteins. Gene accession numbers corresponded to the detected proteins are available in database provided by the Pathosystems Resource Integration Center (PATRIC). The genes with PATRIC IDs fig|1612.50. peg.2355, fig|1612.50.peg.2356 and fig|1612.50.peg.2357 are selected as candidates.

Furthermore, we obtained phosphorylated sinigrin (Supplementary Fig. S2) using $\beta$-glucoside kinase (BglK) from Klebsiella pneumoniae ATCC23357, as the phosphorylation activity on the 6 ' carbon of $\beta$-thioglucosides has been previously reported ${ }^{19}$. We evaluated and compared the glucosidase activity of $C f \mathrm{PbgS}$ for sinigrin and phosphorylated sinigrin using cell-free extracts of a strain expressing CfPbgS, namely, Lc. lactis pNZ7021-pbgS. AITC production was detected only in the reactions using phosphorylated sinigrin (Supplementary Fig. S3). These results are consistent with the putative pathway, which assumes that sinigrin is degraded following transport into cells and undergoes simultaneous phosphorylation, which is carried out by CfPttS and CfPbgS, as presented in Fig. 3B.

\section{Discussion}

In this study, we isolated $\mathrm{Cb}$. farciminis $\mathrm{KB} 1089$ as an important sinigrin-degrading lactic acid bacterium. Moreover, we identified two proteins $(C f \mathrm{PttS}$ and $C f \mathrm{PbgS})$ as the enzymes that are responsible for sinigrin degradation. Our results suggest that PTS mediates substrate import and that phosphorylation is involved in the GSL metabolism of $\mathrm{Cb}$. farciminis KB1089. To date, three bacterial enzymes or genes associated with GSL metabolism have been identified: from Citrobacter WYE1, E. coli and B. thetaiotaomicron VIP-5482. GH3 family $\beta$-O-glucosidase was isolated as a GSL-degrading enzyme from the soil isolate Citrobacter WYE1 ${ }^{20}$. Another enzymatic mechanism that involves four gene products in B. thetaiotaomicron VIP-5482 has been proposed ${ }^{21}$. The metabolic pathway is initiated by the $\mathrm{NAD}^{+}$-dependent oxidation of the sugar moiety, which is followed by hydrolysis and concomitant reduction using $\mathrm{NADH}^{21}$. In addition, it was also found that 6-phospho- $\beta$-glucosidase is involved in GSL metabolism in E. coli strain O157:H722. By differential proteomics on E. coli VL8, a glucose-specific PTS was shown to be induced by sinigrin, which provides some evidence that a phosphorylation step is important for the GSL hydrolysis ${ }^{23}$. These reports indicate that phosphorylation of the glucose moiety through PTS is required for GSL metabolism in these E. coli strains. Thus, the GSL-metabolizing mechanism of $C b$. farciminis KB1089 appears to be similar to that of E. coli rather than Citrobacter WYE1 and B. thetaiotaomicron VIP-5482.

In lactic acid bacteria, some PTS transporters for sugar substrates, such as monosaccharide, disaccharide and sugar alcohol, were found. Moreover, PTS-mediated metabolic pathways of glycosylated aromatic compounds (amygdalin, esculin and salicin) in Lb. acidophilus NCFM have been recently reported ${ }^{24}$. Lb. acidophilus NCFM metabolizes $\beta$ - $(1,6)$-diglucoside and $\beta$-glucoside consisting of mono- or bicyclic aromatic rings conjugated with a glycosyl moiety via PTS, but not sinigrin (allyl- $\beta$-thioglucoside $)^{24}$. This enzymatic mechanism appears to be 
A

$\beta$-glucoside specific IIB, IIA, IIC PTS components (CfPttS)

6-phospho- $\beta$ glucosidase (CfPbgS)
Hypothetical protein (CfNuks)

\begin{tabular}{|c|c|c|}
\hline pttS & pbgs & nukS \\
\hline
\end{tabular}

\section{$\longmapsto$}

$1 \mathrm{~kb}$

B

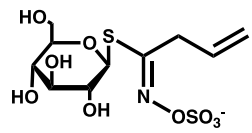

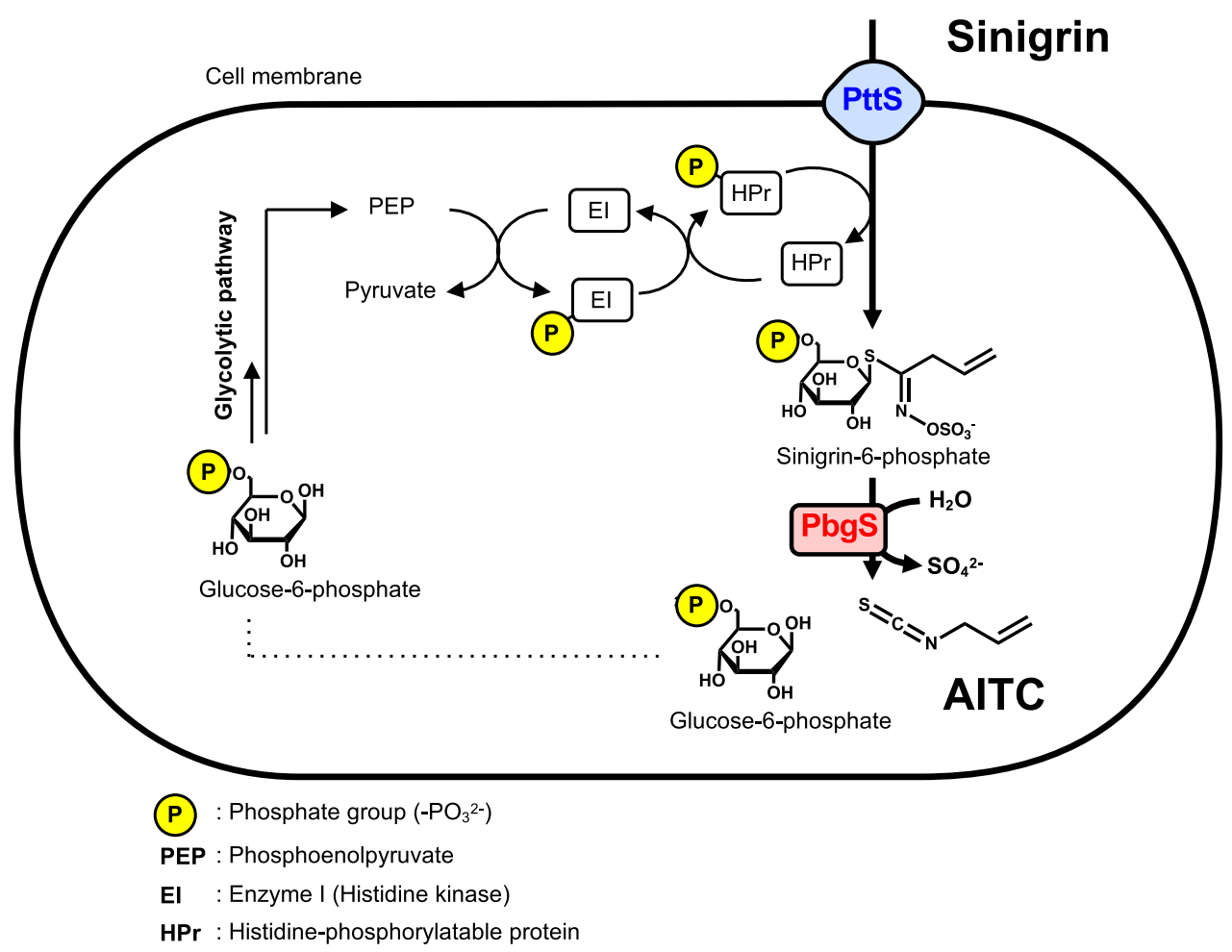

Figure 3. The gene cluster encoding the candidate proteins found in the genome of $C b$. farciminis KB1089 (A) and the putative pathway of sinigrin degradation (B).

similar to that of sinigrin degradation catalyzed by $C f \mathrm{PttS}$ and $C f \mathrm{PbgS}$, whereas the target specificity (i.e., gene regulation and substrate recognition) appears to be different.

InterPro analysis revealed that the CfPttS consists of three components (IIA, IIB and IIC) (Table S4). The amino acid sequence exhibited $41 \%$ identity with the $\beta$-glucoside-specific IIABC component (encoded by LBA0275) in $L b$. acidophilus $\mathrm{NCFM}^{24}$, which contributes to the metabolism of plant glucoside that has small aromatic aglycones. CfPttS does not exhibit high global homology with the $N$-acetyl glucosamine-specific PTS component IIBCA or a glucose-specific PTS component in E. coli VL8 ${ }^{23}$ that has been suspected to be involved in GSL metabolism, whose coding gene is observed in the genomes of the other GSL-degrading strains, O157:H7 and Nissle $1917^{10,22}$. According to PTS-mediated carbohydrate-metabolizing mechanisms, the CfPttS is assumed to enable the transfer of a phosphoryl group to sinigrin from versatile PTS components (EI and HPr). Appropriate arrangement of these components seems important for a successful phosphoryl relay. Destruction of this arrangement by membrane disruption may cause the loss of the sinigrin-degrading activity of cell-free extracts, as observed in $\mathrm{Cb}$. farciminis KB1089 in this study and in other GSL-degrading bacteria in previous studies ${ }^{9,10}$.

CfPbgS belongs to the GH1 family (Table S4), which also contains plant and aphid myrosinase $\mathrm{e}^{25-27}$ and the 6-phospho- $\beta$-D-glucosidases (encoded in bglA and $a s c B$ ) of E. coli O157:H722. CfPbgS exhibits high identities with BglA and AscB of E. coli O157:H7 (48\% and 50\%, as presented in Fig. 5). However, CfPbgS has a low identity $(<20 \%)$ with a bacterial myrosinase from Citrobacter WYE1, which belongs to the GH3 family ${ }^{20}$. Putative 

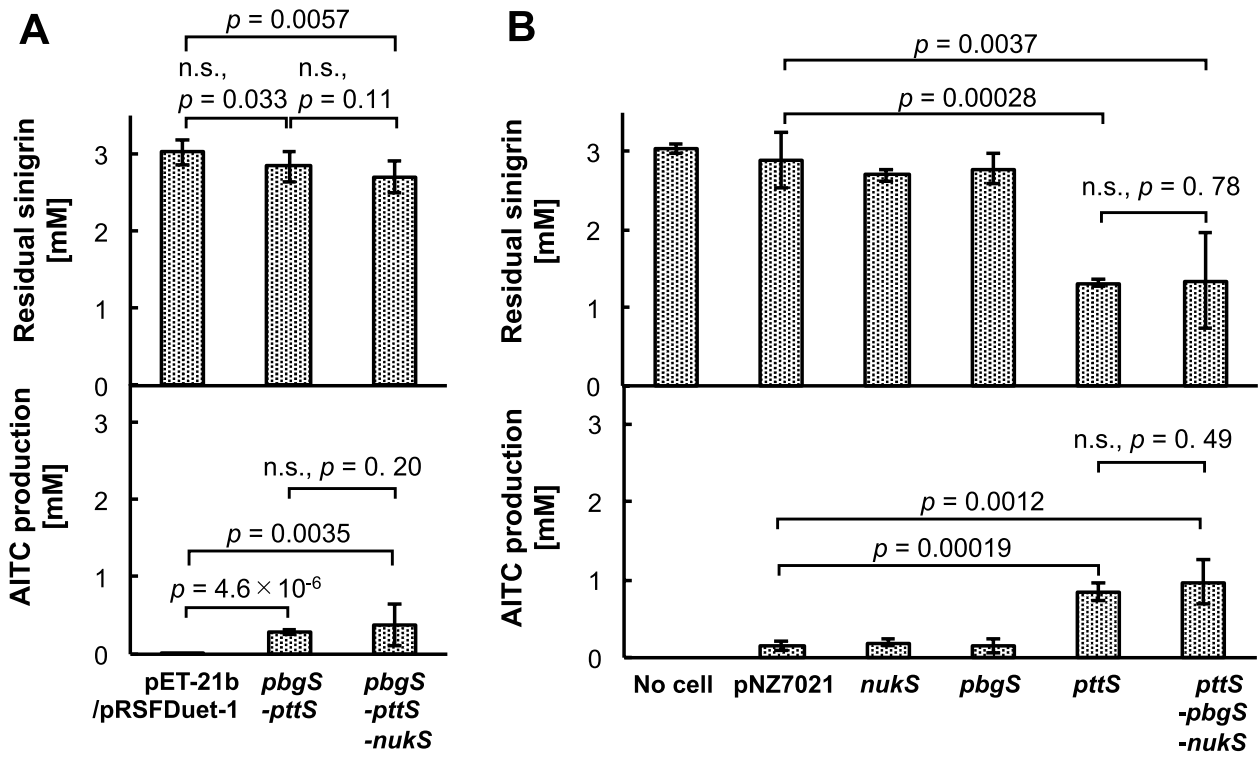

Figure 4. Sinigrin-degrading activity of transformed E. coli or Lc. lactis in the resting cell reactions using washed cells. (A) Amounts of residual sinigrin and produced AITC in the reaction mixtures using transformed E. coli. 'pET-21b/pRSFDuet-1' indicates the vector control strain; ' $p b g S-p t t S$ ' indicates the strain pET21-pbgS/ pRSFDuet-pttS and 'pbgS-pttS-nukS' indicates the strain pET21-pbgS/pRSFDuet-pttS-nukS. (B) Amounts of residual sinigrin and produced AITC in the reaction mixtures using transformed Lc. lactis. 'No cell' indicates a reaction solution without cells; 'pNZ7021' indicates the vector control strain; ' $n u k S$ ' indicates the strain pNZ7021-nukS; 'pbgS' indicates the strain pNZ7021-pbgS; ' $p t t S$ ' indicates the strain pNZ7021-pttS; 'pttS-pbgS$n u k S$ ' indicates the strain pNZ7021-pttS-pbgS-nukS. Data represent mean values $(\mathrm{n}=3)$. Error bars indicate $95 \%$ CI. Student's t-test was performed for statistical analysis. n.s., no significant difference.

\section{A}

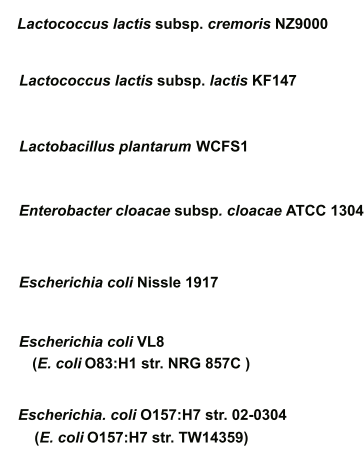

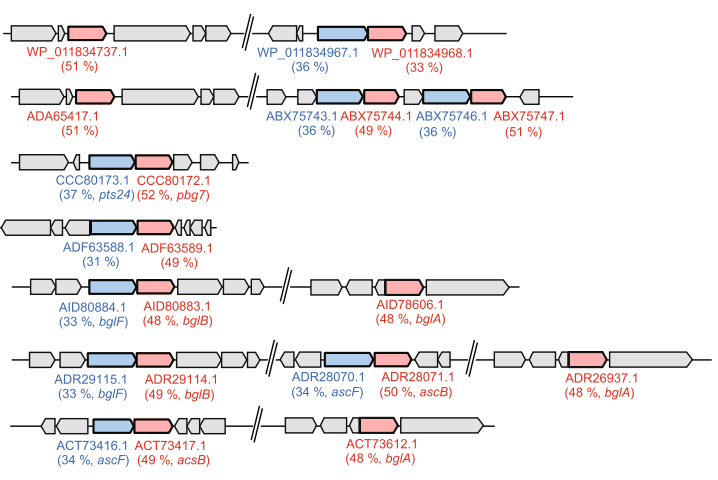

B

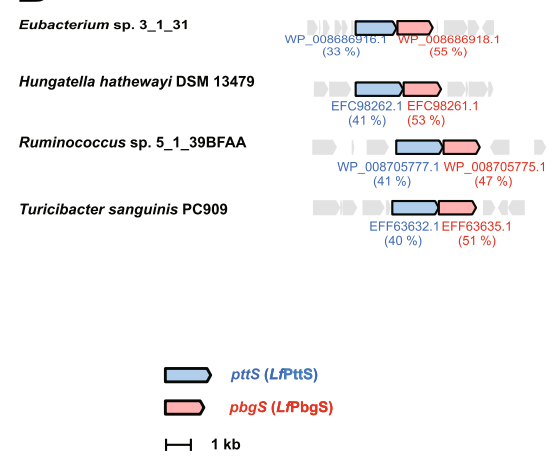

Figure 5. Distribution of gene clusters containing $p t t S$ and $p b g S$ homologues in bacteria. Genes encoding homologues of $C f \mathrm{PttS}$ and $\mathrm{CfPbgS}$ are indicated in blue and red, respectively. The NCBI accession number assigned to each gene encoding a CfPttS or CfPbgS homologue and its sequence identity obtained by BLASTP analysis as well as its gene symbol are presented below its coding region. Sequence identities are presented in brackets below the coding regions. (A) The homologues of $p t t S$ and $p b g S$ in GSL-degrading bacteria. GSLdegrading activities of all bacteria listed above were confirmed in the present study or in previous studies ${ }^{15-17}$. The strains written in parenthesis were used as genome reference in the previous study. (B) The homologues of $p t t S$ and $p b g S$ in gut bacteria. All bacteria listed are gut bacteria whose genomic sequences were obtained by metagenomic sequencing of gut microbiome in the Human Microbiome Project ${ }^{18}$.

myrosinase activity by the 6-phospho- $\beta$-glucosidase from E. coli O157:H7, together with the GSL-induced activation of a glucose PTS in E. coli $\mathrm{VL}^{23}$, has led to the suggestion that glucose moiety phosphorylation may be a prerequisite for GSL hydrolysis in these E. coli strains and in Cb. farciminis KB1089.

We found gene clusters containing homologues of $p t t S$ and $p b g S$ in the genomes of other GSL-degrading bacteria $^{15-17}$, including E. coli strains isolated as human gut bacteria and human associated bacteria (Fig. 5A). However, no homologues were observed in B. thethaiotaomicron ${ }^{21}$. Furthermore, the homologue of $p t t S$ and $p b g S$ were found in other gut bacteria, whose genomes obtained by metagenomic sequencing of gut microbiome in the Human Microbiome Project ${ }^{18}$, such as Eubacterium sp. 3_1_31 and Hungatella hathewayi DSM 13479 which 
had not yet been reported as GSL-degraders (Fig. 5B). For further understanding of GSL metabolism in gut bacteria and lactic acid bacteria, a functional analysis of these homologous genes is underway. To the best of our knowledge, this is the first study that identified novel proteins involved in GSL degradation in lactic acid bacteria.

Together with this finding, proteome analysis also revealed that two NAD-dependent deacetylases, enzymes that catalyze protein deacetylation, are strongly induced in sinigrin containing medium (Table 1). Protein acetylation/deacetylation is a hot topic in recent years because it is an evolutionarily conserved post-translational modification that affects enzyme activity, metabolic flux, and many important biochemical processes, but the regulatory mechanisms have not been well characterized in bacteria ${ }^{28}$. Focusing on these deacetylases may lead to a better understanding of the response mechanisms of lactic acid bacteria to the presence of bioactive phytochemicals such as sinigrin or AITC.

\section{Methods}

Chemicals. Sinigrin was purchased from Sigma-Aldrich (St. Louis, MO, USA) and AITC from Wako Pure Chemicals (Osaka, Japan). All the other chemicals utilized in this study were of analytical grade and are commercially available.

Culture media. De Man, Rogosa and Sharpe (MRS) broth (Difco, BD, Franklin Lakes, NJ, USA) containing $2 \%$ dextrose and basal solution ( $1 \%$ proteose peptone No. $3 ; 1 \%$ beef extract; $0.5 \%$ yeast extract; $0.1 \%$ polysorbate $80 ; 0.2 \%$ ammonium citrate; $0.5 \%$ sodium acetate; $0.01 \%$ magnesium sulphate; $0.005 \%$ manganese sulphate; $2 \%$ dipotassium phosphate) were adjusted to $\mathrm{pH} 6.5$ with $\mathrm{HCl}$ and used for lactic acid bacteria cultivation. Four modified MRS (mMRS) media (G10S6-mMRS, S3-mMRS, G3S3-mMRS, and G3-mMRS) were utilized to analyze the activity of lactic acid bacteria on sinigrin degradation. G10S6-mMRS contained $10 \mathrm{mM}$ glucose, $6 \mathrm{mM}$ sinigrin, and the basal solution. S3-mMRS contained $3 \mathrm{mM}$ sinigrin and the basal solution. G3S3-mMRS contained $3 \mathrm{mM}$ glucose, $3 \mathrm{mM}$ sinigrin, and the basal solution. G3-mMRS contained $10 \mathrm{mM}$ glucose and the basal solution. For the cultivation of $E$. coli transformants, Luria-Bertani (LB) medium containing $1 \%$ tryptone, $0.5 \%$ yeast extract, and $1 \% \mathrm{NaCl}$ was used. For the cultivation of $L c$. lactis transformants, modified M17 (mM17) medium obtained by supplementation of $30 \mathrm{mM}$ glucose and $0.1 \mathrm{mg} \mathrm{mL}^{-1}$ catalase (from bovine liver) to M17 broth (Difco, BD, Franklin Lakes, NJ, USA) was utilized.

Bacterial strains, plasmids, and primers. The bacterial strains, plasmids, and primers utilized in this study are presented in Supplementary Tables S1-S3.

Screening of GSL-metabolizing lactic acid bacteria. Glycerol stocks of laboratory stock lactic acid bacteria, isolated from human feces, animal feces or pickles were inoculated in $10 \mathrm{~mL}$ of MRS broth and were cultivated at $30^{\circ} \mathrm{C}$ without shaking for $24 \mathrm{~h}$. Culture medium $(100 \mu \mathrm{L})$ was added to $10 \mathrm{~mL}$ of fresh MRS broth and was cultivated at $30^{\circ} \mathrm{C}$ without shaking for $24 \mathrm{~h}$. Moreover, culture medium $(50 \mu \mathrm{L})$ was added to $5 \mathrm{~mL}$ of S3-mMRS medium and further cultivated at $30^{\circ} \mathrm{C}$ without shaking for $120 \mathrm{~h}$. The culture medium was centrifuged at $12,000 \times \mathrm{g}$ for $20 \mathrm{~min}$, and $600 \mu \mathrm{L}$ of the obtained supernatant was transferred to a $1.5-\mathrm{mL}$ plastic tube. An identical volume of $30 \%(\mathrm{w} / \mathrm{v})$ trichloroacetic acid (TCA) solution was added to the tube, mixed by inversion, placed on ice for $30 \mathrm{~min}$ and then centrifuged at $12,000 \times \mathrm{g}$ for $20 \mathrm{~min}$. The obtained supernatant was filtered with a membrane filter with a pore size of $0.22 \mu \mathrm{m}$ and analyzed by high-performance liquid chromatography (HPLC). For detailed analytical conditions of HPLC analysis see Supplementary Materials and Methods.

Bacterial species identification. Selected strain KB1089 was identified by $16 \mathrm{~S}$ rRNA and phenylalanyltRNA synthetase $(p h e S)$ gene $^{29}$ sequencing. The detailed methods were provided in Supplementary Materials and Methods.

Time-course evaluation of bacterial growth, $\mathrm{pH}$, glucose and sinigrin concentration in $\mathrm{Cb}$. farciminis KB1089 culture. Cb. farciminis KB1089 was inoculated from a glycerol stock into $5 \mathrm{~mL}$ of MRS broth, cultivated at $30^{\circ} \mathrm{C}$ without shaking for $24 \mathrm{~h}$, and subcultured in fresh MRS broth under the same condition for $24 \mathrm{~h}$. The subculture medium $(350 \mu \mathrm{L})$ was inoculated into $35 \mathrm{~mL}$ of G10S6-mMRS medium and cultivated at $30^{\circ} \mathrm{C}$ without shaking. Then, $1.2 \mathrm{~mL}$ of the culture was sampled at different time points, of which $100 \mu \mathrm{L}$ aliquots were utilized to measure the optical density at $550 \mathrm{~nm}\left(\mathrm{OD}_{550}\right)$ and to determine $\mathrm{pH}$ using a portable $\mathrm{pH}$ meter (Model B212, Horiba, Kyoto, Japan). The remaining $1 \mathrm{~mL}$ of culture was centrifuged and treated with TCA solution, as described above, after which the concentrations of sinigrin and glucose were determined via HPLC and using a Biosensor BF-5i (Oji Scientific Instruments, Amagasaki, Japan), respectively. The experiments were performed in triplicate, and the averages of three separate experiments that were reproducible within $10 \%$ were presented in Fig. 2A.

Cloning of nukS, pbgS, and pttS in E. coli and Lc. lactis. Detailed methods for preparation of genomic DNA, extraction of plasmid DNA, DNA sequence analysis, PCR amplification, and purification of PCR products and cloning of $n u S, p b g S$ and $p t t S$ in E. coli and in Lc. lactis were provided in Supplementary Materials and Methods.

Preparation of washed cell pellets. For preparation of washed cells of $\mathrm{Cb}$. farciminis KB1089, a glycerol stock was inoculated into $10 \mathrm{~mL}$ of MRS broth in glass test tubes $(16.5 \times 125 \mathrm{~mm})$, and was cultivated at $28^{\circ} \mathrm{C}$ with shaking $\left(120\right.$ strokes $\left.\mathrm{min}^{-1}\right)$ for $16 \mathrm{~h}$. Furthermore, $100 \mu \mathrm{L}$ of the culture was added to $10 \mathrm{~mL}$ of either 
G3S3-mMRS or G3-mMRS medium and cultivated at $28^{\circ} \mathrm{C}$ with shaking $\left(120\right.$ strokes $\left.\mathrm{min}^{-1}\right)$ for $7 \mathrm{~h}$. After confirming glucose consumption in the culture medium using a Glucose CII-Test Wako Kit (Wako Pure Chemicals Ind., Osaka, Japan), cells were harvested by centrifugation at $1500 \times g$ for $10 \mathrm{~min}$, and pellets were washed twice with $0.85 \%(\mathrm{w} / \mathrm{v}) \mathrm{NaCl}$.

For preparation of washed cells of E. coli transformants, they were grown at $37^{\circ} \mathrm{C}$ with shaking at 150 strokes $\mathrm{min}^{-1}$ in $5 \mathrm{~mL}$ of LB medium containing $34 \mu \mathrm{g} \mathrm{mL} \mathrm{m}^{-1}$ of chloramphenicol and antibiotics corresponding to the resistance-conferring genes present in the indicated harbored vectors $\left(30 \mu \mathrm{g} \mathrm{mL} \mathrm{L}^{-1}\right.$ of kanamycin for E. coli pET28$n u k S$, E. coli pET28-pbgS, E. coli pET28-pttS and E. coli pRSF-pttS-nukS, and $100 \mu \mathrm{g} \mathrm{mL} \mathrm{m}^{-1}$ ampicillin together with $30 \mu \mathrm{g} \mathrm{mL} \mathrm{m}^{-1}$ kanamycin for E. coli pET21-pbgS/pRSF-pttS and E. coli pET21-pbgS/pRSF-pttS-nukS, respectively) in $12 \mathrm{~mL}$ culture tubes (Thermo Fisher Scientific, Waltham, MA, USA). About $50 \mu \mathrm{L}$ of each overnight culture was added to $5 \mathrm{~mL}$ of LB medium containing the appropriate antibiotics. Isopropyl $\beta$-D-1-thiogalactopyranoside (IPTG) was added to a final concentration of $0.1 \mathrm{mM}$ upon incubation at $37^{\circ} \mathrm{C}$ with shaking at 150 strokes min $^{-1}$ for $2.5 \mathrm{~h}$, and cells were cultivated at $20^{\circ} \mathrm{C}$ with shaking at 120 strokes $\mathrm{min}^{-1}$ for $16 \mathrm{~h}$. The cells were harvested by centrifugation at $1500 \times g$ for $10 \mathrm{~min}$, washed twice with $0.85 \%(\mathrm{w} / \mathrm{v}) \mathrm{NaCl}$.

For preparation of washed cells of Lc. lactis transformants, they were grown under anaerobic conditions (using the AnaroPack, Mitsubishi Gas Chemical, Tokyo, Japan) at $30^{\circ} \mathrm{C}$ for $18 \mathrm{~h}$ in $5 \mathrm{~mL}$ of mM17 medium supplemented with $10 \mu \mathrm{g} \mathrm{mL} \mathrm{m}^{-1}$ of chloramphenicol in glass test tubes $(16.5 \times 150 \mathrm{~mm})$. About $250 \mu \mathrm{L}$ of each culture medium was added to $10 \mathrm{~mL}$ of an identical fresh medium. After incubation (under anaerobic conditions at $30^{\circ} \mathrm{C}$ for $7 \mathrm{~h}$ ), the cells were harvested by centrifugation at $1500 \times g$ for $20 \mathrm{~min}$, washed twice with $0.85 \%(\mathrm{w} / \mathrm{v}) \mathrm{NaCl}$.

Resting cell reactions using washed cells of Cb. farciminis KB1089, E. coli, and Lc. lactis transformants. The washed cell pellets were resuspended in $1 \mathrm{~mL}$ of reaction solution containing $3 \mathrm{mM}$ sinigrin in $20 \mathrm{mM}$ potassium phosphate buffer (KPB, pH 6.5) and incubated at $28^{\circ} \mathrm{C}$ with shaking $\left(120\right.$ strokes $\left.\mathrm{min}^{-1}\right)$ for $16 \mathrm{~h}$. The reaction mixtures were centrifuged at $1500 \times \mathrm{g}$ for $10 \mathrm{~min}$. To detect sinigrin, the supernatants were analyzed by HPLC. Conversely, to detect AITC, $0.6 \mathrm{~mL}$ of the supernatants was transferred to a glass test tube, and an identical volume of hexane, including $25 \mathrm{nM}$ benzyl propionate as the internal standard, was added to the sample. The sample was then mixed by vortexing for $1 \mathrm{~min}$ and centrifuged at $1500 \times g$ for $10 \mathrm{~min}$. The aliquot corresponding to the hexane layer was filtered with a membrane filter (Merck Millipore, Darmstadt, Germany) with $0.45 \mu \mathrm{m}$ pore size and was analyzed by gas chromatography-mass spectrometry (GC-MS). For detailed analytical conditions of GC-MS analysis see Supplementary Materials and Methods.

Synthesis and analysis of sinigrin-6-sinigrin. Detailed methods of enzymatic synthesis of sinigrin6-phosphate by BglK, extraction of phosphorylated sugar compounds and detection of phosphorylated sinigrin by high-performance ion chromatography-high-resolution tandem mass spectrometry (HPIC-HRMS/MS) analysis were provided in Supplementary Materials and Methods.

Reaction using the cell-free extracts of Lc. lactis transformants. Washed cells of Lc. lactis pNZ7021-pbgS were suspended in $1 \mathrm{~mL}$ of $100 \mathrm{mM}$ MES (pH 6.5) and disrupted using Insonator $201 \mathrm{M}$ Ultrasonic Oscillator (Kubota, Osaka, Japan) at $9 \mathrm{kHz}, 180 \mathrm{~W}, 0^{\circ} \mathrm{C}$ for four cycles of $5 \mathrm{~min}$ each. The supernatants obtained following the removal of cell debris by centrifugation at $8000 \times \mathrm{g}$ for $20 \mathrm{~min}$ were used as the cell-free extracts. The cell-free extracts $(450 \mu \mathrm{L})$ were mixed with $50 \mu \mathrm{L}$ of $0.3 \mathrm{mg} \mathrm{mL}^{-1} \mathrm{Ba}^{2+}$ salts containing phosphorylated sugar compounds, obtained as described in Supplementary Materials and Methods, in $100 \mathrm{mM}$ MES (pH 6.5) and incubated at $28{ }^{\circ} \mathrm{C}$ with shaking at 120 strokes $\mathrm{min}^{-1}$ for $1.5 \mathrm{~h}$. Subsequently, the produced AITC was extracted and analyzed as described above.

Draft genome sequencing. Draft genome sequences of Cb. farciminis KB1089, LMG9189, NRIC0416 and NRIC0417 were determined by the whole-genome shotgun strategy using 454 pyrosequencing in the GS Junior Benchtop System (Roche, Basel, Switzerland). The draft genome sequences of Cb. farciminis KB1089, LMG9189, NRIC0416 and NRIC0417 were obtained with 67, 67, 112 and 166 contigs, respectively.

Quantitative proteomic analysis. Washed cells of $\mathrm{Cb}$. farciminis KB109 prepared using G3S3-mMRS and G3-mMRS media, as described above, were used for proteomics as induced and non-induced cells, respectively. For detailed preparation procedures for quantitative proteomic analysis see Supplementary Materials and Methods. LC-MS/MS analysis was conducted using an LC (Ultimate 3000; Thermo Scientific, Waltham, MA, USA) -MS-MS (LTQ Velos Mass Spectrometer, Thermo Scientific, Waltham, MA, USA) system equipped with a long monolithic capillary column. Tryptic digests were separated by reversed-phase chromatography using a monolithic silica capillary column $(200 \mathrm{~cm} \text { long, } 0.1 \mathrm{~mm} \mathrm{ID})^{30}$ at the flow rate of the two eluents: eluent A, $0.1 \%(\mathrm{v} / \mathrm{v})$ formic acid; eluent $\mathrm{B}, 80 \%$ acetonitrile containing $0.1 \%(\mathrm{v} / \mathrm{v})$ formic acid. The gradient started with $5 \%$ eluent B, increased to $45 \%$ eluent B for 600 min, further increased to $95 \%$ eluent B to wash the column for $140 \mathrm{~min}$, returned to the initial condition, and then held for re-equilibration of the column. Separated analytes were detected using a mass spectrometer with a full-scan range of $350-1500 \mathrm{~m} / \mathrm{z}$. The analysis program was set to automatically analyze the top 10 most intense ions observed in the MS scan for data-dependent acquisition. An electrospray ionization voltage of $2.4 \mathrm{kV}$ was directly applied to the LC buffer end of the chromatography column by using a MicroTee (Upchurch Scientific, Lake Forest, IL, USA). The ion transfer tube temperature was set to $300^{\circ} \mathrm{C}$. Then, data analysis was conducted using Proteome Discoverer version 2.1 (Thermo Scientific, Waltham, MA, USA). The data were filtered with a cut-off $q$-value $\leq 0.01$, corresponding to a $1 \%$ false discovery rate on a spectral level. Protein identification was performed using the Mascot algorithm against the protein database of $\mathrm{Cb}$. farciminis KB1089 obtained from draft genome sequence. 


\section{Data availability}

The genome nucleotide sequence data of the Cb. farciminis strains are available in the DNA Data Bank of Japan (DDBJ) database under the accession numbers BHYW01000001-BHYW01000067 for KB1089, BHYX01000001BHYX01000067 for LMG9189, BHYY01000001-BHYY01000112 for NRIC0416, and BHYZ01000001BHYZ01000166 for NRIC0417. The genomic features database of $\mathrm{Cb}$. farciminis KB1089 are available in the PATRIC (the Pathosystems Resource Integration Center) database with Genome ID 1612.50. The proteomic data were deposited in the Japan ProteOme Standard Repository/Database (jPOST) ${ }^{31}$ with the accession number PXD011820. The $p t t S, p b g S$ and $n u k S$ gene sequences were deposited in DDBJ under accession numbers LC422123, LC422124 and LC422125, respectively.

Received: 12 February 2021; Accepted: 18 November 2021

Published online: 09 December 2021

\section{References}

1. Zhang, Y., Talalay, P., Cho, C. G. \& Posner, G. H. A major inducer of anticarcinogenic protective enzymes from broccoli: Isolation and elucidation of structure. Proc. Natl. Acad. Sci. USA 289(6), 399-2403 (1992).

2. Higdon, J. V., Delage, B., Williams, D. E. \& Dashwood, R. H. Cruciferous vegetables and human cancer risk: Epidemiologic evidence and mechanistic basis. Pharmacol. Res. 55(3), 224-236 (2007).

3. Gross-Steinmeyer, K. et al. Sulforaphane- and phenethyl isothiocyanate-induced inhibition of aflatoxin B1-mediated genotoxicity in human hepatocytes: Role of GSTM1 genotype and CYP3A4 gene expression. Toxicol. Sci. 116(2), 422-432 (2010).

4. Ahn, Y. H. et al. Electrophilic tuning of the chemoprotective natural product sulforaphane. Proc. Natl. Acad. Sci. USA 107(2), 9590-9595 (2010).

5. Elbarbry, F. \& Elrody, N. Potential health benefits of sulforaphane: A review of the experimental, clinical and epidemiological evidences and underlying mechanisms. J. Med. Plants Res. 5(4), 473-484 (2011).

6. Herr, I. \& Büchler, M. W. Dietary constituents of broccoli and other cruciferous vegetables: Implications for prevention and therapy of cancer. Cancer. Treat. Rev. 36(5), 377-383 (2010).

7. Elfoul, L. et al. Formation of allyl isothiocyanate from sinigrin in the digestive tract of rats monoassociated with a human colonic strain of Bacteroides thetaiotaomicron. FEMS Microbiol. Lett. 197, 99-103 (2001).

8. Sakurama, H. et al. $\beta$-Glucuronidase from Lactobacillus brevis useful for baicalin hydrolysis belongs to glycoside hydrolase family 30. Appl. Microbiol. Biotechnol. 98, 4021-4032 (2014).

9. Llanos Palop, M., Smiths, J. P. \& Brink, B. T. Degradation of sinigrin by Lactobacillus agilis strain R16. Int. J. Food Microbiol. 26(2), 219-229 (1995).

10. Narbad, A. \& Rossiter, T. J. Gut glucosinolate metabolism and isothiocyanate production. Mol. Nutr. Food Res. 62, 1-10 (2018).

11. Postma, P. W., Lengeler, J. W. \& Jacobson, G. R. Phosphoenolpyruvate:carbohydrate phosphotransferase systems of bacteria. Microbiol. Rev. 57, 543-594 (1993).

12. Henrissat, B. \& Davis, G. Structural and sequence-based classification of glycoside hydrolases. Curr. Opin. Struct. Biol. 7, 637-644 (1997).

13. Saraste, M., Sibbald, P. R. \& Wittinghofer, A. The P-loop: A common motif in ATP- and GTP-binding proteins. Trends Biochem. Sci. 15, 430-434 (1990).

14. Ettlinger, M. G. \& Lundeen, A. J. The structures of sinigrin and sinalbin; an enzymatic rearrangement. J. Am. Chem. Soc. 78(16), 4172-4173 (1956).

15. Luciano, F. B., Belland, J. \& Holley, R. A. Microbial and chemical origins of the bactericidal activity of thermally treated yellow mustard powder toward Escherichia coli O157:H7 during dry sausage ripening. Int. J. Food Microbiol. 145(1), 69-76 (2011).

16. Mullaney, J. A., Kelly, W. J., McGhie, T. K., Ansell, J. \& Heyes, J. A. Lactic acid bacteria convert glucosinolates to nitriles efficiently yet differently from Enterobacteriaceae. J. Agric. Food Chem. 61, 3039-3046 (2013).

17. Luang-In, V. et al. The metabolism of methylsulfinylalkyl- and methylthioalkyl-glucosinolates by a selection of human gut bacteria. Mol. Nutr. Food Res. 58, 875-883 (2014).

18. NIH HMP Working Group, Peterson, J., Garges, S., Giovanni, M., McInnes, P., Wang, L., Schloss, J. A., Bonazzi, V., McEwen, J. E., Wetterstrand, K. A., Deal, C., Baker, C. C., Di Francesco, V., Howcroft, T. K., Karp, R. W., Lunsford, R. D., Wellington, C.R., Belachew, T., Wright, M., Giblin, C., David, H., Mills, M., Salomon, R., Mullins, C., Akolkar, B., Begg, L., Davis, C., Grandison, L., Humble, M., Khalsa, J., Little, A. R., Peavy, H., Pontzer, C., Portnoy, M., Sayre, M. H., Starke-Reed, P., Zakhari, S., Read, J., Watson, B. \& Guyer, M. The NIH Human Microbiome Project. Genome Res. 19, 2317-2323 (2009).

19. Thompson, J., Lichtenthaler, F. W., Peters, S. \& Pikis, A. $\beta$-glucoside kinase (BglK) from Klebsiella pneumonia. Purification, properties, and preparative synthesis of 6-phospho- $\beta$-D-glucosides. J. Biol. Chem. 277(37), 34310-34321 (2002).

20. Albaser, A. et al. Discovery of a bacterial glycoside hydrolase family 3 (GH3) $\beta$-glucosidase with myrosinase activity from a Citrobacter strain isolated from soil. J. Agric. Food Chem. 64(7), 1520-1527 (2016).

21. Liou, C. S. et al. A metabolic pathway for activation of dietary glucosinolates by a human gut symbiont. Cell 180, 717-728 (2020).

22. Cordeiro, R. P., Doria, J. H., Zhanel, G. G., Sparling, R. \& Holley, R. A. Role of glycoside hydrolase genes in sinigrin degradation by E. coli O157: H7. Int. J. Food Microbiol. 205, 105-111 (2015).

23. Luang-In, V., Narbad, A., Cebeci, F., Bennett, M. \& Rossiter, J. T. Identification of proteins possibly involved in glucosinolate metabolism in L. agilis R16 and E. coli VL8. Protein J. 34(2), 135-146 (2015).

24. Theilmann, M. C. et al. Lactobacillus acidophilus metabolizes dietary plant glucosides and externalizes their bioactive phytochemicals. MBio 8(6), e01421-e1517 (2017).

25. Bones, A. M. \& Rossiter, J. T. The enzymic and chemically induced decomposition of glucosinolates. Phytochemistry 67(11), 1053-1067 (2006).

26. Pontoppidan, B., Ekbom, B., Eriksson, S. \& Meijer, J. Purification and characterization of a non-plant myrosinase from the cabbage aphid (Brevicoryne brassicae), a brassica herbivore. Eur. J. Biochem. 268(4), 1041-1048 (2001).

27. Jones, A. M., Winge, P., Bones, A. M., Cole, R. \& Rossiter, J. T. Characterization and evolution of a myrosinase from the cabbage aphid Brevicoryne brassicae. Insect Biochem. Mol. Biol. 32(3), 275-284 (2002).

28. Liu, M. et al. Bacterial protein acetylation and its role in cellular physiology and metabolic regulation. Biotechnol. Adv. 53, 107842 (2021).

29. Scheirlinck, I. et al. Lactobacillus crustorum sp. Nov., isolated from two traditional Belgian wheat sourdoughs. Int. J. Syst. Evol. Microbiol. 57, 1461-1467 (2007).

30. Morisaka, H. et al. Profile of native cellulosomal proteins of Clostridium cellulovorans adapted to various carbon sources. AMB Express 2(1), 37 (2012).

31. Okuda, S. et al. jPOSTrepo: An international standard data repository for proteomes. Nucl. Acid. Res. 45, D1107-D1111 (2017) 


\section{Acknowledgements}

This work was partially supported by JSPS KAKENHI Grant Number JP 15H02441 and 16K14884. This paper is partially supported by the New Energy and Industrial Technology Development Organization (NEDO). The results were obtained by using microbial resources developed in the NEDO project (J.O.). It is also partially supported by the Ministry of Health and Welfare of Japan and Public/Private R\&D Investment Strategic Expansion Program: PRISM (J.O.). We thank S. Aburaya, K. Esaka, and M. Ueda for their technical assistance with quantitative proteomic analysis.

\section{Author contributions}

H.W., R.U., S.K., K.O., Y.A., H.S, and J.O. planned experiments; H.W., R.U., K.O., Y.A., H.M., M.T., and Y.I. performed experiments; H.W., R.U., S.K., K.O., Y.A., H.M., M.T., Y.I., T.B., W.A., and J.O. analyzed data; H.W., R.U., S.K., and J.O. wrote the paper.

\section{Competing interests}

The authors declare no competing interests.

\section{Additional information}

Supplementary Information The online version contains supplementary material available at https://doi.org/ 10.1038/s41598-021-03064-7.

Correspondence and requests for materials should be addressed to J.O.

Reprints and permissions information is available at www.nature.com/reprints.

Publisher's note Springer Nature remains neutral with regard to jurisdictional claims in published maps and institutional affiliations.

(c) Open Access This article is licensed under a Creative Commons Attribution 4.0 International License, which permits use, sharing, adaptation, distribution and reproduction in any medium or format, as long as you give appropriate credit to the original author(s) and the source, provide a link to the Creative Commons licence, and indicate if changes were made. The images or other third party material in this article are included in the article's Creative Commons licence, unless indicated otherwise in a credit line to the material. If material is not included in the article's Creative Commons licence and your intended use is not permitted by statutory regulation or exceeds the permitted use, you will need to obtain permission directly from the copyright holder. To view a copy of this licence, visit http://creativecommons.org/licenses/by/4.0/.

(C) The Author(s) 2021 